\title{
Discriminative whisking in the head-fixed rat: optoelectronic monitoring during tactile detection and discrimination tasks
}

\author{
MICHAEL A. HARVEY, ROBERTO BERMEJO and H. PHILIP ZEIGLER
}

Biopsychology Program, Hunter College, CUNY, New York, NY 10021, USA

\begin{abstract}
We compared whisking movement patterns during acquisition of tactile detection and object discrimination under conditions in which (a) head movements are excluded and (b) exposure to tactile discriminanda is confined to the large, moveable vibrissae (macrovibrissae). We used optoelectronic instrumentation to track the movements of an individual whisker with high spatio-temporal resolution and a testing paradigm, which allowed us to dissociate performance on an "indicator" response (lever pressing) from the rat's "observing" responses (discriminative whisking). We analyzed the relation between discrimination performance and whisking movement patterns in order to clarify the process by which the indicator response comes under the stimulus control of information acquired by the rat's whisking behavior. Whisking patterns over the course of task acquisition differed with task demands. Acquisition of the Detection task was correlated with modulation of only one whisking movement parameter - total number of whisks emitted, and more whisking was seen on trials in which the discriminandum was absent. Discrimination between a sphere and cube differing in size and texture was correlated with a reduction in whisk duration and protraction amplitude and with a shift towards higher whisking frequencies. Our findings confirm previous reports that acquisition of tactile discriminations involves modulation by the animal of both the amount and the type of whisking. In contrast with a previous report (Brecht et al., 1997), they indicate that rats can solve tactile object detection and discrimination tasks (a) using only the large, motile mystacial vibrissae (macrovibrissae) and (b) without engaging in head movements. We conclude that the functional contribution of the macrovibrissae will vary with the nature of the task and the conditions of testing.
\end{abstract}

Key words: whisking, detection, discrimination, kinematics, head fixation

\section{Introduction}

Much of our knowledge of sensory processing mechanisms is derived from studies of neural or behavioral responses to stimuli passively applied to sensory surfaces. In nature, however, animals seek out and actively engage stimuli, focusing on biologically relevant features (Willis and Arbas, 1997). Once such a feature has been located it may be subjected to progressively more detailed examination by observing responses (Zeigler and Wyckoff, 1961)-responses which provide exposure to the relevant stimuli and mediate encoding of critical stimulus properties. Appropriately organized "observing response" patterns could potentially maximize the resolution of specific stimulus properties and facilitate selection of appropriate goaldirected behaviors. For example, humans, when asked to discriminate among objects with respect to their haptic properties (e.g., hardness, volume, or shape) solely by touch, typically use hand movements specific for each property (Lederman and Klatzky, 1987). Perception may thus be viewed as a process with two sensorimotor dimensions: "acting to sense" (exploration) and "sensing to act" (discrimination). The present report examines the interaction of these two dimensions during discriminative behavior in the rat, using novel methods of discrimination training and response monitoring.
The rodent's mystacial vibrissa (whisker) system provides a useful preparation for the study of neurobehavioral mechanisms mediating both exploration and discrimination. The vibrissa motor system generates whisking movements, which may be modulated in amplitude, frequency, and velocity. The resulting somatosensory input patterns are then used to guide the movements of diverse effectors (neck, jaw, limbs), and, recursively, to control the whisking movements themselves. The vibrissae are involved in exploration, in spatial mapping of the environment, and in the detection, localization and discrimination of objects (Vincent, 1912; Hutson and Masterton, 1986; Carvell and Simons, 1990; Brecht et al., 1997). Vibrissal movement patterns function as a sensorimotor interface between sensory inputs and exploratory and discriminative behaviors and the relation is relatively direct.

The vibrissa system generates two classes of whisking behavior: a large amplitude "exploratory" pattern at about $6-9 \mathrm{~Hz}$, which modulates, upon contact (Welker, 1964) into a "discriminative" whisking pattern of lower amplitude and higher frequencies (Carvell and Simons, 1990). During "discriminative" whisking, the large lateral vibrissae emit whisking patterns whose kinematic properties (bandwidth, protraction amplitude) are related specifically to surface features of the discriminanda (Carvell and Simons, 1995). These investigators 
report that successful performance on texture discrimination tasks was generally correlated with amount of time spent whisking, and success on specific tasks was correlated with the development of specific whisking "strategies". Conversely, adult rats subjected to clipping of vibrissae during development were impaired in the solution of a tactile discrimination task, and the impairment was correlated with the absence of whisking frequencies in the 6-12 Hz range (Carvell and Simons, 1996). These workers suggest that the rat's whiskers operate as a high-resolution tactile sensing organ, generating movement patterns which may be modulated to meet the functional requirements of discriminative tasks.

This conclusion has been questioned in a recent report by Brecht et al. (1997). These investigators distinguish, on morphological grounds, between the "long, laterally oriented macrovibrissae" and the "shorter, more numerous and more rostral microvibrissae". Based upon this morphological analysis and the results of behavioral studies, they suggest that the two groups of sensory hairs have quite different functions. They argue that the primary function of the macrovibrissae is to provide information about object location and that object recognition is mediated by the microvibrissae.

The testing paradigms and tasks used by the two laboratories make quite different demands upon the vibrissa system, and these differences may account for the differing conclusions of the two studies. However, the currently available evidence provides support for both positions. Previous studies of the mystacial vibrissae are clearly consistent with a tactile detection and/or spatial localization function (Vincent, 1912; Hutson and Masterton, 1986), while the Carvell and Simons (1990, 1995) studies convincingly demonstrate a role for the macrovibrissae in discrimination behavior. On the other hand, the motor innervation of the macro- and microvibrissae differs significantly. The follicular innervation of the macrovibrissae permits movements of individual whiskers, in addition to those associated with movements of the entire mystacial pad. This follicular innervation is lacking in the microvibrissae (Dorfl, 1985). In this respect, they resemble the vibrissae of the sea-lion, which are not actively "whisked" during discrimination, but are swept passively across the discriminanda by lateral movements of the animal's head (Dehnhardt, 1994; Dehnhardt and Ducker, 1996). Inspection of videographic records (pers. commun. H. Kyriazi and D. Simons, 1999) indicates that discriminative whisking behavior in rats is associated with both whisker and head movements. The extent to which such head movements play a role in tactile discrimination by rodents is unknown, since they have not been controlled in any previous study.

Indeed, studies of discriminative whisking have been constrained by significant problems of experimental control and measurement. The paradigms typically used (e.g., gap-jumping, maze-running) are incompatible with control of the sensory surface engaging the stimulus, and cannot exclude a contribution by head movements. They involve considerable experimenter/animal interaction and may require the use of visual occluders. Moreover, videography has a relatively low spatio-temporal resolution, relative to the frequency and amplitude range of whisking movements, and the labor-intensive nature of videographic analysis reduces the amount of data available for (e.g., trial-by-trial) analysis.

One aim of the present study is to examine the relation between whisking behavior and discriminative performance under conditions in which (a) head movements are excluded and (b) exposure to the tactile stimuli is confined to the macrovibrissae. A second is to compare whisking movement patterns using tasks making differing demands upon the mystacial vibrissae but differing from the texture discriminations used in previous studies. An ancillary goal was the development of methods and instrumentation for the high-resolution monitoring of discriminative whisking behavior under controlled conditions. To facilitate experimental control and measurement of discriminative whisking we have (a) utilized a head-fixed preparation, (b) developed instrumentation for the high-resolution optoelectronic monitoring of individual whisker movement trajectories, with all other whiskers present, (c) used tactile discrimination paradigms which facilitate dissociation of "indicator" and "observing" responses during discriminative behaviors, and (d) developed software for the rapid and efficient (computerassisted) acquisition and analysis of whisking data. We have used these procedures to examine patterns of whisking behavior associated with the acquisition of two different whisking-mediated tasks. The first task involved simply detection of the presence of a single tactile discriminandum; the second, required discrimination between a pair of objects differing along several different dimensions (size, shape, texture).

Here we report (a) that head-fixed rats can solve tactile detection and object discrimination tasks, using only the macrovibrissae, and (b) confirm previous reports (Carvell and Simons, 1995) demonstrating significant correlations between successful performance and specific whisking movement parameters.

\section{Materials and methods}

Subjects

The data presented in this paper were obtained from eight rats, both males and females, of the Long-Evans and Sprague-Dawley strains, ranging in age from 3 months to a year. (A number of additional animals were used but discarded, either because they lost their head mounts or failed to acquire and/or maintain stable performance in the operant lever-pressing phase of the study.) Animals were housed individually under a $12: 12$ reversed lightdark cycle. During testing, subjects were water-deprived and 
maintained at $80-85 \%$ of free-feeding weight by a $23.5 \mathrm{~h}$ water deprivation schedule with food available ad libitum.

\section{Surgery}

Subjects were anesthetized with ketamine/xylazine $(100 \mathrm{mg} / \mathrm{kg}$, i.p. $/ 5.5 \mathrm{mg} / \mathrm{kg}$, i.m.) for placement of a dental cement head mount which contained a mounting screw (Small Parts \#Q-TSB-632-12) embedded in its central portion (Bermejo et al., 1996).

\section{Apparatus}

Training and testing were carried out in a soundproof chamber (interior dimensions $80 \times 60 \times 60 \mathrm{~cm}$ ). The chamber contained a gravity-driven water delivery system, a white-noise generator, a stepping motor, an optoelectronic monitoring device (PAS $11 \mathrm{H}$, laser micrometer, Hama Laboratories, Palo Alto, CA) and a CCD camera. Illumination for closed-circuit video observations was provided by an LED emitting infrared illumination at a wavelength $(950 \mathrm{~nm})$ beyond the visible spectrum of the rat (Rosenberger and Ernest, 1970; Messing, 1972). Stimulus presentation, water delivery, data collection and storage were controlled by a 486 PC using customized software written in QuickBasic.

For testing, the animal's body movements were constrained and its head fixed in position using a V-shaped Plexiglas restrainer containing a metal bracket to which the head mounting screw is fixed (Bermejo et al., 1996). A microswitch attached to the platform of the restrainer serves as an operant lever. During testing, the restrainer was fixed in place in the testing chamber and stimuli were rotated into position by the stepping motor. Figure 1A schematically illustrates the experimental arrangements. (Note: For clarity, only a few whiskers on each side are shown. However, although we monitored only the movements of a single whisker (C-1) in each animal, all whiskers were present on both sides during testing.)

\section{Optoelectronic monitoring of individual vibrissa movements}

Whisker movements were monitored along a plane that includes the rostro-caudal axis and is perpendicular to the whisker pad using an optoelectronic system (laser-emitter and detector) which has been described in detail elsewhere (Bermejo et al., 1998). The 2,496 sensors in the detector are arranged in a $28 \mathrm{~mm}$ linear array. Interruption of the emitted beam by the shadow of a whisker generates a voltage shift in the subset of shaded sensors (CCDs). Whisker movement trajectories produce successive displacements in the position of that voltage shift, which are linearly related to whisker position. A comparator circuit identifies the successive positions of voltages above a preset threshold and outputs the data to a microprocessor for computation and display of the trajectory. To monitor an individual whisker trajectory with all other whiskers present, a light $(3-6 \mathrm{mg})$ rectangular $(1 \times 1 \times 18 \mathrm{~mm})$ foam marker is attached to the selected whisker with one end close to but not touching the base of the whisker. This increases the marker's detectability with respect to surrounding vibrissae without significantly affecting whisking kinematics (Bermejo et al., op. cit.). Figure 1B illustrates both the principle of the monitoring system and the rationale for our calibration procedure.

\section{Calibration}

In order to transform data on sensor locations into a record of angular whisker positions, a calibration procedure is carried out for each animal at the start of each recording session. The whisker is positioned manually at $90^{\circ}$ from the horizontal, i.e., perpendicular to the animal's snout. The detector is placed so that the whisker shaft intercepts the CCD array at its mid point, and at a fixed distance $(c .10 \mathrm{~mm})$ from the whisker base. The position of that CCD (e.g., 1-2,496) intersected by the shadow of the whisker at its initial (i.e., $90^{\circ}$ ) position is recorded. The angular displacement of the vibrissa may then be calculated using the formula $\arctan (\theta)=$ Opposite/Adjacent, where the opposite is the distance moved along the CCD array and the adjacent is some known distance. (Note: Since the whisker moves in an arc, the point on the vibrissa shaft that interrupts the beam at the beginning of the trajectory will not be identical with the point interrupting the beam at its end. This could result in an overestimation of the distance traveled by the whisker. To compensate for this we oriented the CCD array in parallel with the animal's face. Since the kinematic properties of the trajectories measured using this procedure were comparable to those reported using videographic methods (see Results), we believe that minor errors introduced by the procedure did not significantly bias the results.)

\section{Behavioral testing}

Operant lever pressing served as the indicator response. Head-fixed animals were initially hand-shaped to press the lever for reinforcement (water, in $40 \mu 1$ aliquots) delivered initially on a Continuous Reinforcement Schedule (CRF). Acquisition of the operant response took place in stages moving from the CRF, through a Fixed Ratio to a Variable Interval schedule, in which reinforcement was delivered for the first response following a predetermined time interval. Detection or discrimination training was initiated when stable, moderate response rates were obtained on a VI 30s schedule. (A variable interval schedule was chosen to provide a stable, moderate rate of responding, as well as to keep the animal from using the water as a discriminant.) Training was conducted in $40 \mathrm{~min}$ daily sessions using a two-component
A

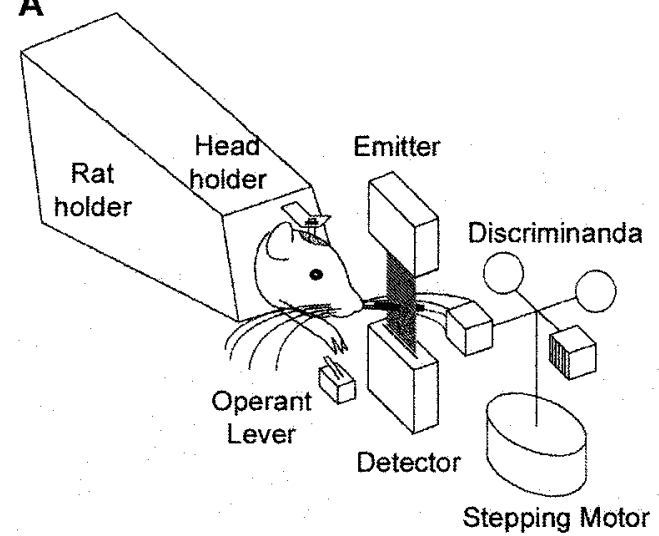

B

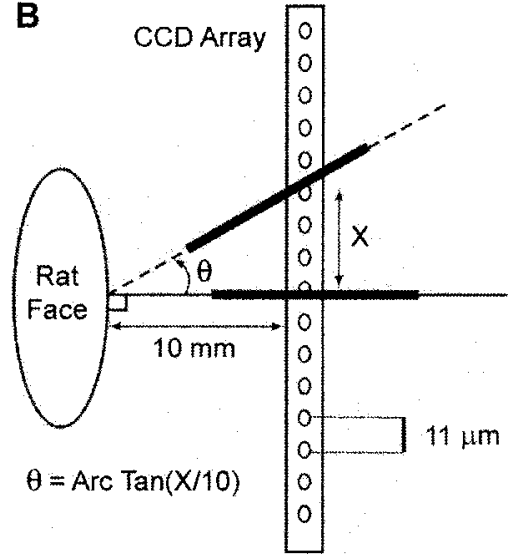

FIGURE 1. (A) Optoelectronic monitoring of whisking during acquisition of a tactile discrimination task. The rat's head is fixed to a metal bar protruding from the top of the restraining device. Its paw rests on a microswitch which records lever presses. A turntable driven by a stepping motor rotates a single discriminandum (sphere/cube) into the rat's "whisking space". The movements of a single whisker (marked to increase its relative detectability) are detected by a laser emitter and an array of CCD detectors. For clarity, only a few whiskers on each side of the face are shown but all whiskers were present during testing. (B) Schematic diagram illustrating both the basic principle of the monitoring system and the rationale of the calibration procedure. Heavy black lines indicate the successive positions of the marked vibrissa during a whisking movement. 
(reinforcement/extinction) schedule in which the presence or absence of a reinforcement was associated with a specific stimulus condition $\left(S+/ S^{-}\right)$. Individual components were created in a quasi-random fashion with no component being shorter than the maximum VI and with equal numbers of each component presented in random order. Trials could end without reinforcement, but reinforcement was available in all $\mathrm{S}+$ trials. These conditions effectively transformed the operant VI schedule into a successive (Go/NoGo) discrimination paradigm.

Subjects were trained either to detect the presence of a $2 \times 2 \times$ $2 \mathrm{~cm}^{3}$ acrylic cube faced with No. 8 sandpaper, or to discriminate between the same acrylic cube and an acrylic sphere, $1 \mathrm{~cm}$ in diameter, i.e., between objects differing in size, surface texture, and shape. In both tasks the stepping motor was used to rotate tactile discriminanda into position parallel to the vibrissae on one side of the snout. The stimuli were positioned such that whisker contact was possible only when the whiskers were protracted, but they were within reach of even low-amplitude whisking movements. In the detection task, subjects were reinforced for lever presses in the presence of the cube $(S+)$ and extinguished in its absence $\left(S^{-}\right)$. In the discrimination paradigm subjects were reinforced for lever pressing in the presence of the sphere $(S+)$ and extinguished in the presence of the cube $\left(\mathrm{S}^{-}\right)$. Under these conditions, it is possible that animals could press at a low rate until delivery of a reinforcement, and then emit a burst of responses, artificially elevating the percentage of correct responses. To provide a performance measure independent of lever pressing, we also recorded the latency from the time of stimulus presentation to the first lever pressing response. However, our primary measure of discrimination performance was the ratio of $\mathrm{S}+/ \mathrm{S}-$ responses, which was calculated at the end of each test session. Criterion performance on the two tasks was defined as $80 \%$ correct responses for three successive sessions. Subjects who achieved criterion were either transferred to a different task or served in one of several control procedures.

\section{Control procedures}

Several features of the experimental setup were designed to insure that solution of the detection and discrimination tasks was mediated by tactile (whisker) inputs, rather than by other sensory cues. Visual cues were eliminated by running the animal in a darkened chamber, illuminated (for video monitoring purposes) by an infrared LED at a wavelength outside the rat's visible spectrum. To control for cues from rotation of the stepping motor which presented the stimuli, the motor had four stopping positions. The four stimulus positions were arranged such that a $90^{\circ}$ rotation of the stepping motor could take the animal into either an $S+$ or $S-$ component, regardless of the current position of the motor. This was done so that the animal could not use differences in the duration of motor operation as an auditory cue. In addition, two animals which had achieved criterion were tested with the stepping motor programmed normally but the discriminanda absent. In three Detection subjects which had met criteria using the left whisker array, several training sessions were carried out in which the discriminanda were presented to the whiskers on the right side of the face to test for transfer. After reacquiring criterion, these animals were placed on a texture discrimination task which required them to discriminate between rough (Norton \#47750/60 grit: Course) and smooth (Norton \#44710/220 grit: Very Fine) surfaces.

\section{Data collection and analysis}

The basic epoch of data selected for analysis was defined by the presentation of the stimulus at the start of each individual trial. Data on lever pressing were saved throughout the extent of each trial. For trials longer than $24 \mathrm{~s}$, data on vibrissa movements were saved for the initial and final $12 \mathrm{~s}$ of the trial-including whisks made during the reinforcement period. The top panel of Figure 2 presents a record of whisking and lever pressing responses recorded during a single trial on the discrimination task. For analysis, the transformed whisking data for each test session were plotted in angular coordinates and displayed on a computer monitor as a plot of whisker position against time. A specially written, cursor-driven program was used to display individual data epochs (Fig. 2, middle panel). Individual whisks were selected for analysis based on their general shape (i.e., a protraction followed by a retraction). A computer algorithm identified critical points, defining the start, peak and end of the whisk (Fig. 2, bottom
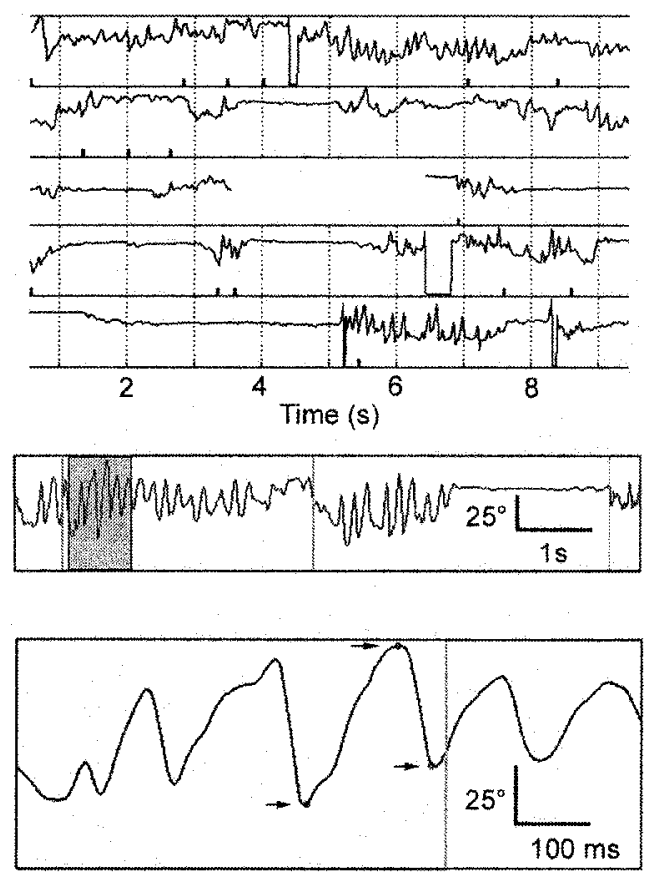

FIGURE 2. Analysis of discriminative whisking movement parameters. Top panel: A record of "whisks" recorded from an identified whisker during a single trial. Ticks at the bottom of each record indicate lever-pressing responses. The interruption of the record represents a period during the middle of the trial when data were not being recorded. Middle panel: A low-resolution view of a $7 \mathrm{~s}$ epoch of individual whisker movements. A cursor-driven analysis program is used to select segments of the record (shaded portion) for analysis. Vertical lines indicate the occurrence of lever presses. Bottom panel: The data segment selected in the middle panel is displayed at higher resolution. Arrows indicate the start, peak and end-point of a selected whisk.

panel), the peak being defined simply as the maximum forward position attained prior to retraction. Whisk duration was defined as the interval between peak protractions and conventional methods were used to calculate the values of other kinematic parameters (e.g., peak amplitude, velocity) as well as the number of whisking responses. Data on numbers of lever presses, latency to first lever press, number of whisking movements and derived kinematic values were saved and exported to a standard spreadsheet program for further computations, graphic presentation and statistical analysis. The values for the kinematic parameters were measured both for total trials $\left(S+/ S^{-}\right)$and (separately) for $S+$ and $S-$ trials. Time series data were analyzed using a Fast Fourier analysis (FFT) of $45 \mathrm{~s}$ blocks of whisking.

\section{Results}

The results presented in this report are based upon an analysis of approximately 50,000 whisking responses (c. 5,000,000 individual data points) obtained from eight rats, over the course of acquisition of a tactile detection or object discrimination task. For each task we present detailed data on performance and whisking behavior for two animals which achieved criterion and one which did not.

\section{Detection: task acquisition}

Five of the seven animals tested reached criterion performance on the detection task within 30 days. Good performance on the task was indicated both by 

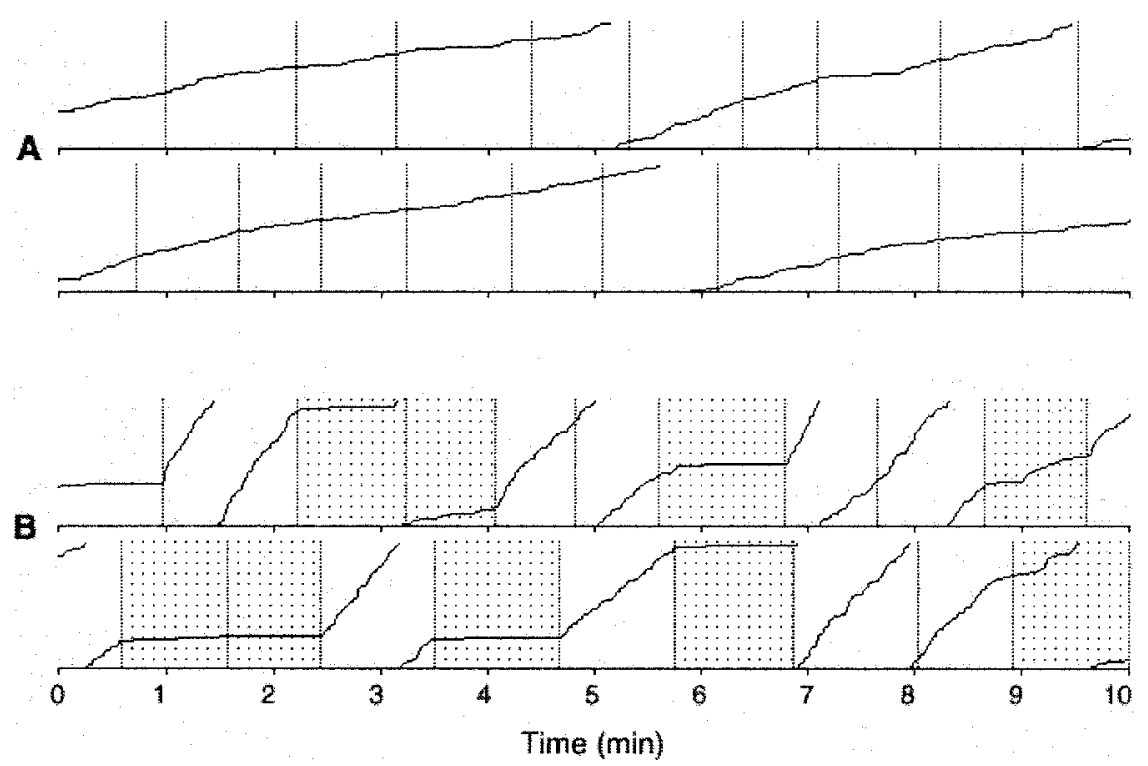

FIGURE 3. Cumulative records of lever pressing for a single representative animal before and after acquisition of a tactile Detection task. In both panels the slope of the curve is proportional to the rate of lever pressing and the cumulative record resets after 70 responses. (A) Cumulative records of lever pressing during the final phase of training on the VI schedule. (B) Responding by the same animal after it has reached criterion on the Detection task. Unshaded/shaded areas represent the S+/S- components of the trial, respectively. Note the rate differences between the two components and the rapid onset (or offset) of responding when the stimulus component changes.

the differential rate of responding on the $\mathrm{S}+$ component of the schedule and by differences in the latency to the first response on a given trial. Figure 3 presents cumulative records of lever pressing on a Variable Interval task (A) prior to the introduction of the discriminandum and (B) after achieving criterion performance on the detection task. Note that lever pressing is stable in the top panel and appears to be under good stimulus control in the discrimination task, with little or no lever pressing on the S- trials (shaded), and an immediate increase in responding at the onset of the $S+$ trial (unshaded).
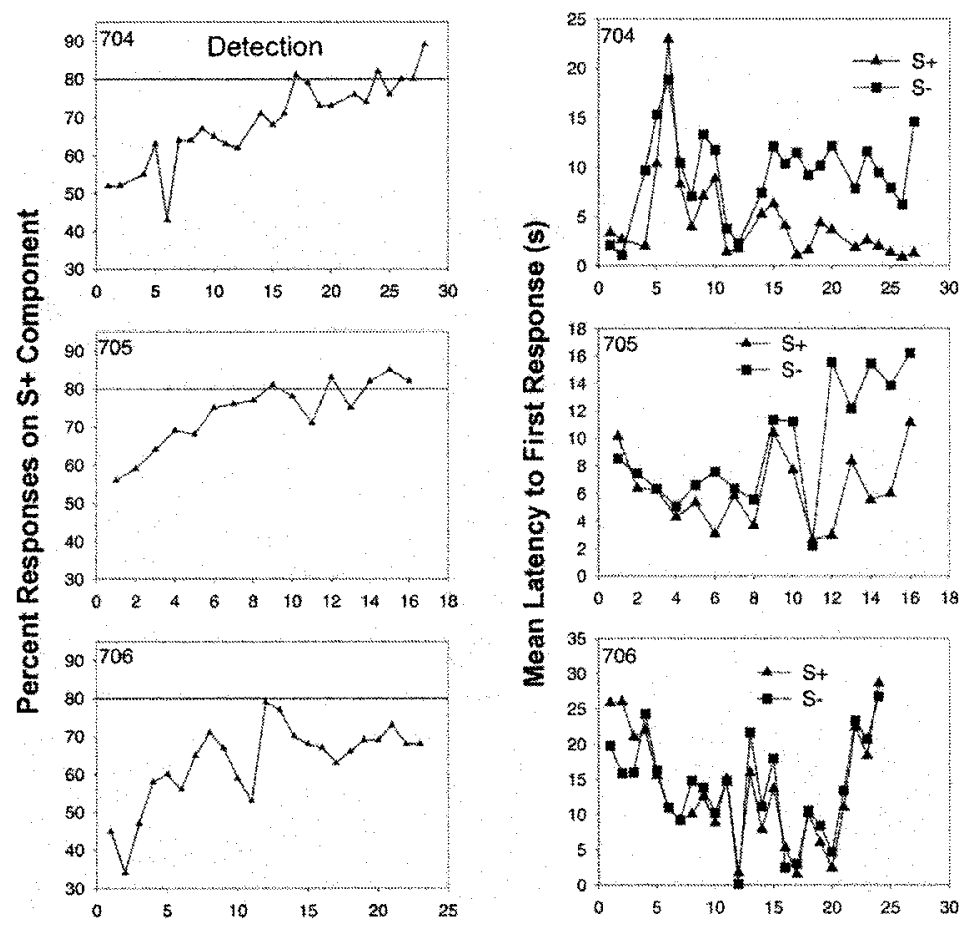

\section{Session}

Figure 4. Relation between latency to first lever press and performance on a tactile Detection task. Data for two animals which achieved criterion $(704,705)$ and a third which did not $(706)$. Left panel: Learning curves based upon lever pressing response rates in the $\mathrm{S}+$ and S- components of the VI schedule. The horizontal line at $80 \%$ indicates the performance criterion. Right panel: Mean latency from the change in the schedule component $\left(\mathrm{S}+/ \mathrm{S}^{-}\right)$to the first lever press for each of the three animals whose performance data are shown in the left panel. 
The left-hand portion of Figure 4 presents learning curves for three representative animals on the Detection task. Two of the animals (704, 705) achieved criterion performance; the third (706), though obviously responding at a higher rate on the $\mathrm{S}+$ component of the schedule, did not. The righthand portion of the figure plots the mean latency to the first lever pressing response in each schedule component. Over the course of task acquisition the two successful animals showed a marked divergence in latencies on the $\mathrm{S}+$ and $\mathrm{S}$ - components which was associated with performance changes during acquisition, but which was not seen in the unsuccessful animal.

\section{Detection: whisking behavior}

To assess changes in whisking behavior accompanying acquisition of the detection task, data on whisk
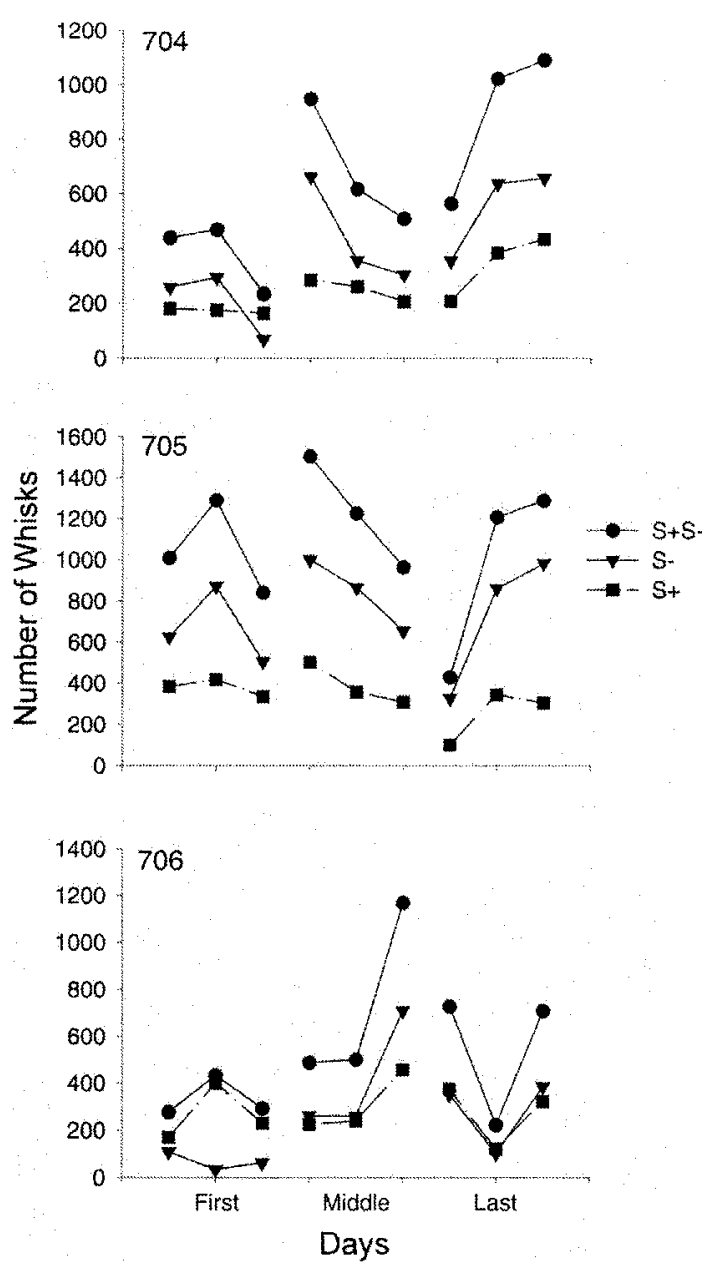

FIGURE 5. Relation between Detection performance and amount of whisking. The figure plots trial blocks from the first, middle, and last three test sessions. Data shown indicate both the total number of whisks emitted in each session $(\mathrm{S}+\mathrm{S}-$ ) and the number of whisks made in the presence $(S+)$ or absence $\left(S^{-}\right)$of the stimulus. Note the divergence over the course of training in the whisking behavior of the animals that achieved criterion ( 704 , 705), and the greater number of whisks on the S-component. No such changes are seen in animal 706, which did not achieve criterion performance. duration, mean protraction amplitude and peak velocity, and number of whisks were analyzed for each of the three rats whose learning curves are presented in Figure 4. For all measures, the analysis was based upon data from the first, middle and last three test sessions. Only one of these measures (number of whisks) varied significantly over the course of training. The two animals achieving criterion performance emitted substantially (30-40\%) more whisks on the $\mathrm{S}-$ trials over the course of task acquisition (Fig. 5). A Fourier analysis of whisking across sessions for each animal did not show any significant differences in whisking frequency related to performance on the detection task.

To relate whisking movement parameters to performance on the indicator (lever pressing) response we constructed a trial-by-trial analysis of the final session of training for all three animals. Correlations were calculated separately for S + and S- trials. Once again, only the amount of whisking was significantly correlated with a performance variable (lever pressing). For rat 704, these correlations were significant $(r=0.55 ; p<0.01)$ only on $\mathrm{S}+$ trials. For rat 705 they were significant for both $S+$ trials $(r=0.74 ; p$ $<0.005)$ and $S-$ trials $(r=0.82 ; p<0.001)$. Lever pressing rates for the last session of rat 706 were so low that meaningful correlations could not be calculated.

\section{Discrimination: task acquisition}

Of the four animals which entered the study, one lost its head mount in the middle of training, two achieved criterion (801 and 825), and one (821) failed to achieve criterion after 2 months of training. Performance data on the discrimination task is presented in Figure 6. Note the striking difference in acquisition time between rats 801 and 825 and that the performance of 821 , though highly variable, remains consistently above chance level for the last month of testing. As with the detection animals, acquisition of the discrimination was associated with a divergence in the latencies to the first lever press only for the successful animals.

\section{Discrimination: whisking behavior}

Acquisition of the object discrimination task was associated with changes in several whisking movement parameters. As in the detection task, the successful animals emitted more whisks (c. 30\%) on $\mathrm{S}-$ trials during the final third of the sessions. All three rats showed a decrease in protraction amplitude from the first to the final session. The difference was highly significant for the two rats that achieved criterion (801: $t=3.62 ; p<0.001 ; 825: t=6.01$; $p<0.001 ; 821: t=2.29 ; p<0.05)$. Both successful animals showed a decreased mean whisking velocity over the course of acquisition, which was due, primarily to a reduction of about $20 \%$ on the 

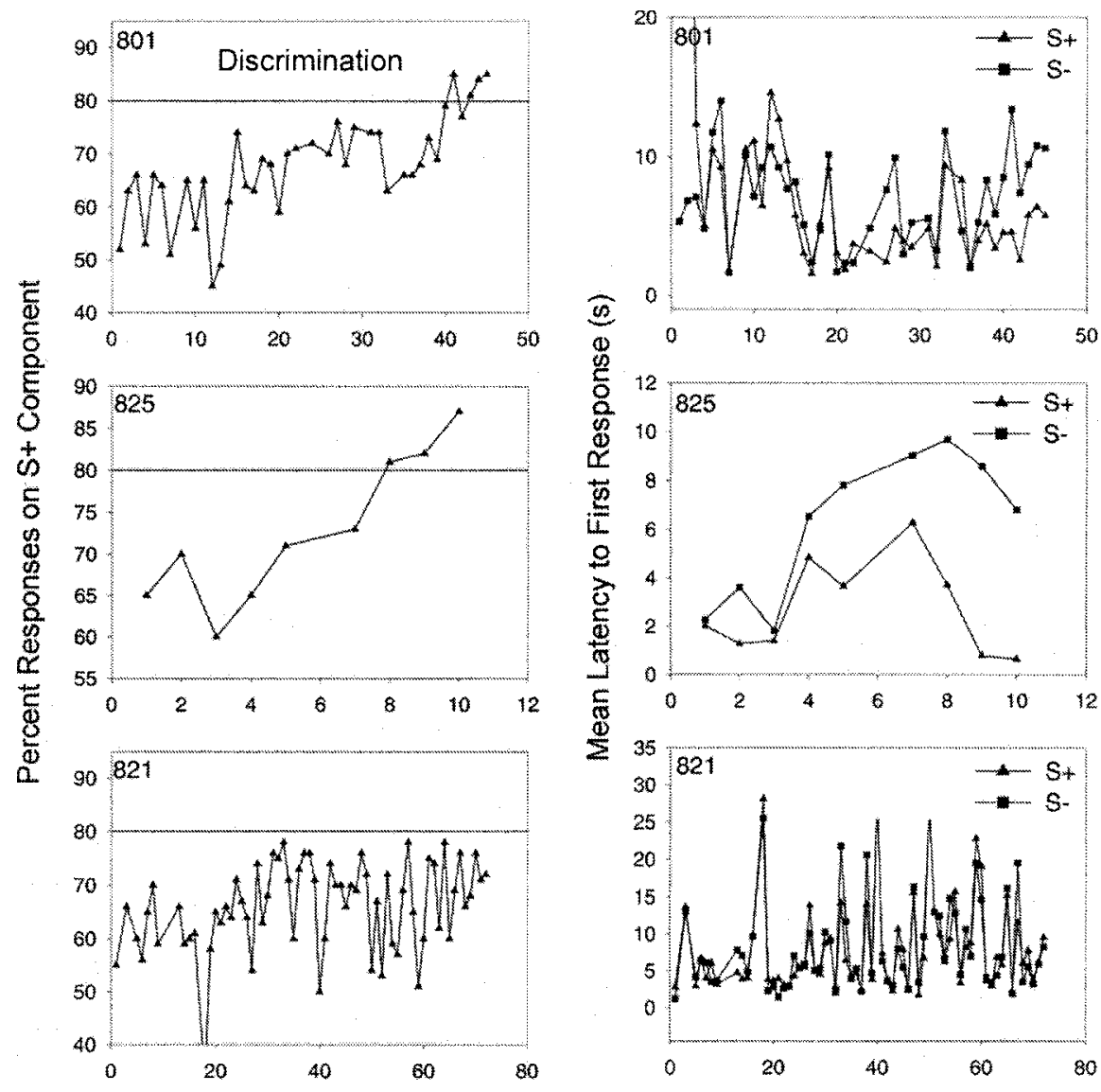

\section{Session}

FIGURE 6. Relation between Discrimination performance and latency to the first lever press. Data for two animals which achieved criterion $(801,825)$ and a third which did not $(821)$. Left panels: Learning curves, based upon lever pressing response rates in the $S+($ sphere) and $\mathrm{S}$ - (cube) components of the VI schedule. The horizontal line at $80 \%$ indicates the performance criterion. Right panel: Mean latency from the change in the schedule component $\left(S+/ S^{-}\right)$to the first lever press. Note the divergence in response latency in 801 and 825 and its absence in 821 .

$\mathrm{S}+$ trials. Moreover, both animals that achieved criterion showed a significant decrease in whisk duration from the first to the final training session (801: $t=3.7$; $p<0.001$; 825: $t=9.7$; $p<0.001$ ). The reduction in whisk duration is consistent with a shift towards higher whisking frequencies in the whisking power spectra of the two animals which achieved criterion performance (801, 825), but not in that of 821, the unsuccessful animal (Fig. 7; Table 1).

A trial-by-trial analysis of the correlations among whisking movement parameters and lever pressing rates was carried out for $S+$ and $S$ - trials during the last test session in all three Discrimination subjects. For rat 801 , correlations were positive and significant on the $\mathrm{S}+$ trials for the number of whisks $(r=0.58$; $p<0.007)$, for whisk durations $(r=0.72 ; p<$ $0.0003)$ and whisk amplitudes $(r=0.50 ; p<0.05)$. For rat 825, they are significant only for a number of whisks $(r=0.57 ; p<0.0009)$. For rat 821, which did not achieve criterion, there are significant negative correlations (on $S+$ trials) between the rate of lever pressing and the duration $(p<0.004)$ and amplitude $(p<0.008)$ of whisking movements.
Figure 8 summarizes the relation between whisking movement parameters (kinematics) and performance over the course of task acquisition for one Detection and one Discrimination animal each of which had attained criterion performance on the task. The figure plots the session means of each of the kinematic variables (duration, amplitude, velocity, number of whisks) as well as a regression line. Significance levels for each kinematic variable were obtained using an ANOVA. Performance on the Detection task is correlated significantly only with the number of whisks emitted, while performance on the Discrimination task is correlated both with

TABLe 1. Area under FFT $\left(\mathrm{O}^{2}\right)$ for high and low frequencies

\begin{tabular}{lccc}
\hline Animal & Session & $0-8 \mathrm{~Hz}$ & $8-15 \mathrm{~Hz}$ \\
\hline 801 & First & 4.39 & 1.52 \\
801 & Last & 4.40 & 4.03 \\
821 & First & 3.62 & 1.59 \\
821 & Last & 4.05 & 1.24 \\
\hline
\end{tabular}



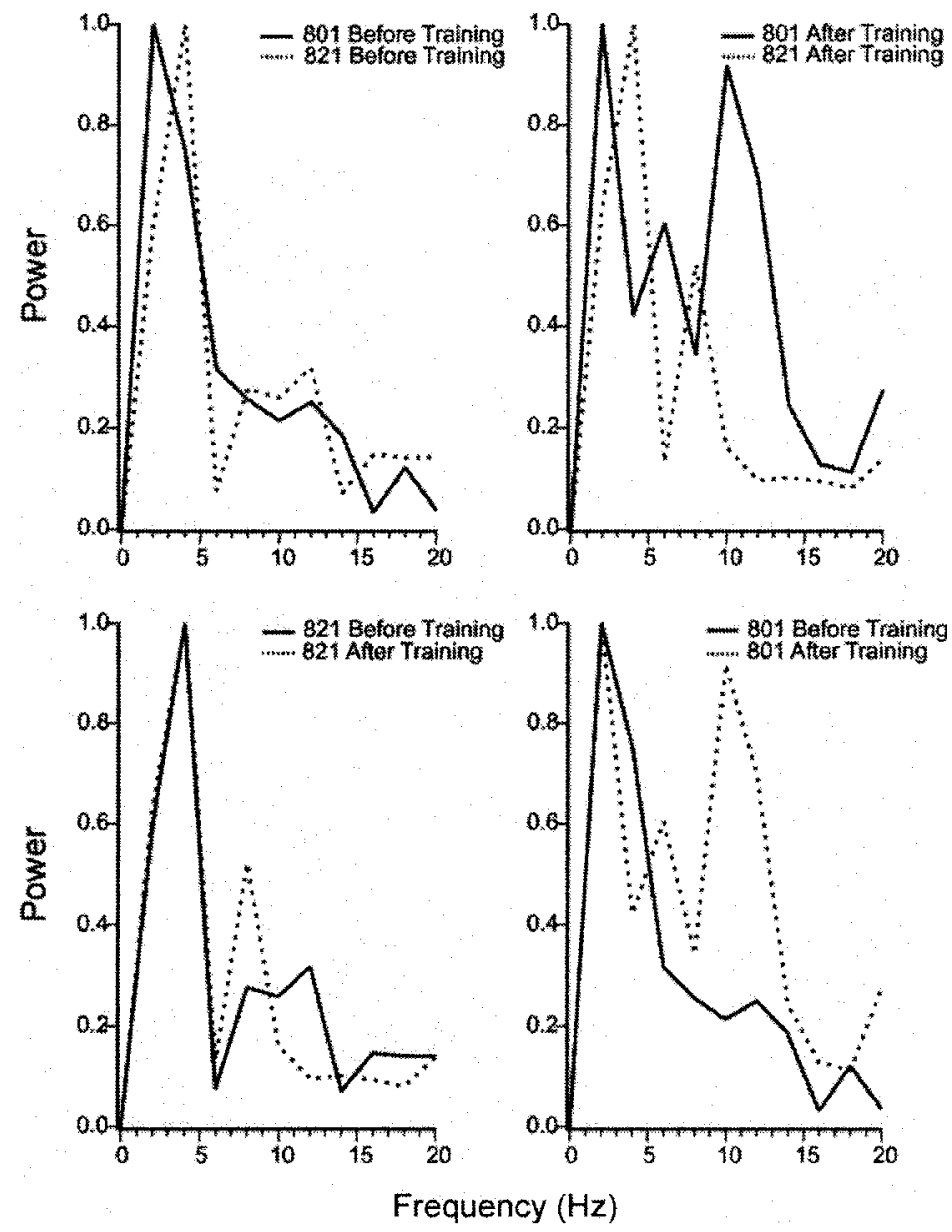

FIGURE 7. Changes in whisking frequency during acquisition of a tactile Object Discrimination. Data for one animal which did (801) and one animal which did not (821) meet criterion on the discrimination task. Power spectra in each panel are based upon a Fourier analysis of whisking on 45 trials for that session. Data for the first training session are compared with that for the final training session. A shift towards higher modal frequencies is evident in the successful, but not in the unsuccessful animal. See also Table 1.
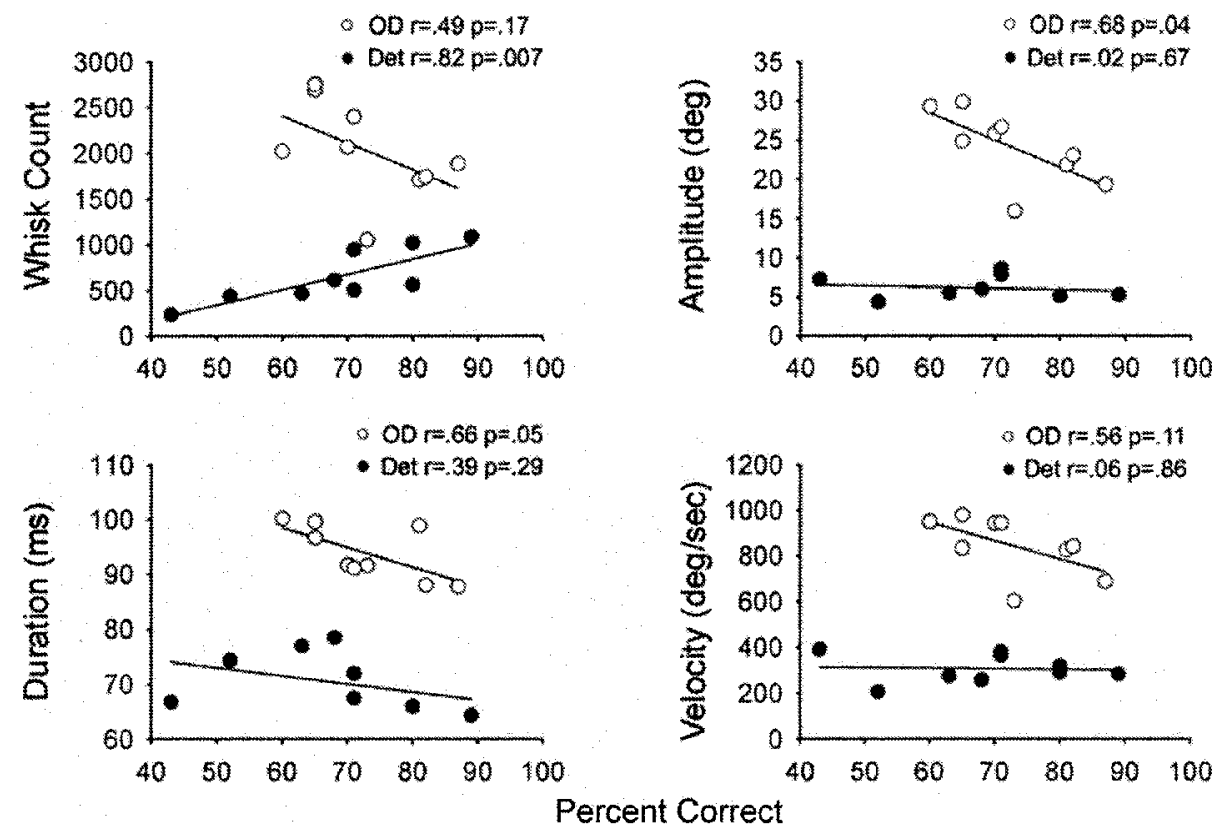

FIGURE 8. Relations among whisking movement parameters and performance during acquisition of tactile Detection and Discrimination tasks. The plots compare the correlations among these parameters for one Detection and one Discrimination animal, each of which achieved criterion performance on the task. In the Detection task, only the correlation with the amount of whisking is significant; in the Discrimination tasks, there are significant correlations for both protraction amplitude and duration. 


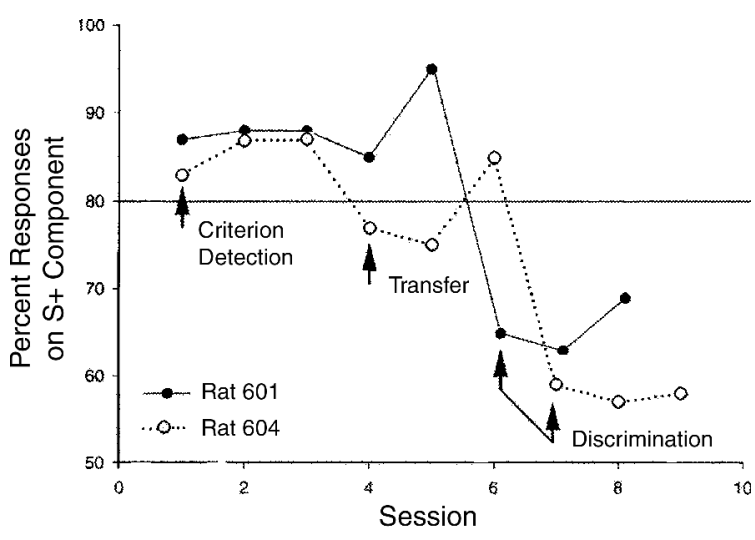

FIGURE 9. Effects of transferring stimulus presentation from the "experienced" to the "naïve" whiskers. Following acquisition of criterion on the Detection task with the whiskers on one side of the face, the stimulus was shifted to the other side. Both animals maintain excellent performance. Shifting to a Rough/Smooth discrimination disrupts performance in subsequent sessions.

shorter whisk durations and smaller protraction amplitudes and there is a (non-significant) trend towards decreased velocity with improved performance.

\section{Control procedures}

In two detection animals that had attained criterion performance, the stimulus was removed from the stepping motor prior to the session, and the trials then proceeded normally. These animals continued to make lever presses during the first few trials and then gradually stopped responding. In three additional animals that had achieved criterion, stimulus presentation was shifted from the whiskers on the left to those on the right. This transfer was followed by an initial decrease in performance, but all three animals regained criterion performance within two sessions. These animals were then shifted to a texture discrimination (rough/smooth). Transfer to the new task was followed by a precipitate drop in differential responding and performance remained at this level for several weeks. Data for two of these "transfer" animals are presented in Figure 9.

\section{Discussion}

We have shown (a) that rats can solve tactile object detection and discrimination tasks using only the large, motile mystacial vibrissae and (b) without engaging in head movements. We have confirmed and extended previous findings (a) that acquisition of discrimination tasks is accompanied by changes in whisking behavior patterns, and (b) that successful performance on the different tasks is associated with changes in different whisking movement parameters (Carvell and Simons, 1995). Our data were obtained in head-fixed, immobilized subjects using a methodology which permits us to track the movements of an individual whisker with high spatio-temporal resolu- tion and in "real" time. Our findings are thus of both substantive and methodological interest.

\section{Methodological considerations}

Our methodology differs from current procedures for the study of discriminative whisking with respect to training paradigm, stimulus control, response measurement and data analysis. Because testing is carried out under infrared illumination, visual occluders are not needed, and the use of an operant "indicator" response minimizes the repeated experimenter/animal interactions associated with jumping stands and mazes. Immobilization and head fixation eliminate confounds related to head movement, and facilitate precise control of stimulus presentation (e.g., to either the right or left whiskers, or exclusively to the macrovibrissae). Head fixation also enables us to use a monitoring system, which provides far higher spatio-temporal resolution than is available with all but the most sophisticated video motionanalysis systems. Moreover, optoelectronic monitoring facilitates rapid and efficient (computer-assisted) acquisition and analysis of whisking data, making it possible to collect data on thousands of whisks (tens of thousands of data points) on large numbers of individual trials, over the long series of training sessions associated with tactile discrimination learning-including both negative and positive trials. (The optoelectronic system does not provide information on whisker/object interactions (e.g., whisker bending) or contact onset and offset (but see Bermejo and Zeigler, 2000).) Finally, the operant discrimination paradigm facilitates dissociation of the rat's "observing responses" (discriminative whisking), from its "indicator" response (lever pressing). It allows us to measure each response class independently and to analyze the process by which the indicator response comes under the stimulus control of information acquired by whisking behavior.

In principle, these methods represent a considerable advance over current procedures, but only if they do not disrupt normal patterns of discriminative whisking or bias data collection in a systematic manner. Put succinctly, do our methods generate data consistent with those obtained by conventional procedures. This appears to be the case. Our data indicate that the relation between discriminative whisking patterns and performance observed in head-fixed animals are (a) systematic and (b) similar to those obtained from unconstrained rats using videographic recording (see below). (Of course, the technique does not distinguish between whisker displacements attributable to the action of follicular (intrinsic) muscles and those reflecting changes in the movement baseline produced by action of the mystacial pad (extrinsic) muscles (Dorfl, 1982).)

Moreover, our results strongly suggest that solution of the detection and discrimination tasks was 
mediated by tactile (whisker) inputs, rather than by extraneous stimuli fortuitously associated with reinforcement. Perhaps the most convincing evidence is the contrast between the rapid reacquisition of criterion performance following presentation of the stimuli to the "naive" whiskers, and the precipitate drop in performance following the shift from the detection to the texture discrimination task (Fig. 10). In the latter task, subjects continued to press the lever but responded non-differentially, i.e., during both $\mathrm{S}+$ and $\mathrm{S}-$ trials. Their persistence in responding in both conditions is consistent with their prior training history, since (on the detection task) lever presses had been reinforced in the presence of a discriminandum and extinguished in its absence. In the texture discrimination task a discriminandum is present on both $\mathrm{S}+$ and $\mathrm{S}-$ trials. The absence of differential responding in this situation (with all other testing conditions constant) is strong evidence that the lever pressing of these animals was controlled by stimulus-reinforcer contingencies associated with vibrissa-mediated tactile inputs from the stimulus objects.

One final methodological consideration is worth noting. In previous studies of tactile localization and discrimination, the rats were initially allowed to palpate the discriminanda with their snouts and the discriminanda were then gradually moved until the indicator response (gap-jumping) was presumably controlled only by the whiskers (Hutson and Masterton, 1986; Carvell and Simons, 1995). Thus the final discrimination performance involves both initial transfer of stimulus control from snout afferents to vibrissa, and subsequent vibrissa-mediated acquisition. The present testing arrangements allow us to track the development of a whisking "strategy", mediated solely by the macrovibrissae, over the course of task acquisition.

\section{Acquisition and performance on the detection and discrimination tasks}

Unlike the "species-typical" jumping/reaching responses of previous studies, the lever pressing response had to be acquired and maintained by the rat prior to the start of discrimination training. Acquisition and maintenance of this "indicator response" was difficult for many animals, probably due to the physical constraints associated with immobilization and head fixation, and males seemed to adapt better to the testing situation than females. In addition to the need for handling and a period of adaptation to the test situation, the size, force requirements, and position of the lever relative to the animal were critical variables. We found, as have others (Welsh, pers. commun., 1998) that initial acquisition of the lever press was facilitated by relatively severe (transient) water deprivation and the use of a successive approximation (shaping) procedure in the initial session(s). A small microswitch, with minimal force requirements, placed horizontally, in front of and parallel with the paw, was effective, but the use of different animal-response configurations and deprivation-reinforcement arrangements might facilitate performance.

Most animals that acquired the response and entered the discrimination phase, performed satisfactorily over prolonged periods of testing. While there was considerable individual variability in the time course of acquisition for both tasks, improvement in performance, on both, was signaled by an increased latency of lever pressing on $\mathrm{S}-$ schedule components, and a decreased latency on $\mathrm{S}+$ trials. Since subjects initially had been trained to press the lever at a steady but moderate rate, these variations in latency during discriminative behavior provide additional evidence for modulation of response rate by the discriminanda.

\section{Whisking kinematics during detection and discrimination}

The two tasks make quite different demands on the vibrissa as an "active touch" system. In the detection task, the animal must simply confirm the presence of an object within the "planar sensory field" formed by the whiskers (Wineski, 1983). This may not require modulation of the basic whisking movement pattern. In the discrimination task the animal has to distinguish the stimulus properties of one object from those of another. Identifying salient features which distinguish the two discriminanda may require the animal to actively engage the object and process the inputs which characterize those features. Differences between the whisking patterns associated with successful performance on the two tasks may thus reflect differing task demands.

Five whisking movement parameters were examined over the course of task acquisition: whisking frequency, the amplitude, velocity and duration of individual whisks, and the amount of whisking. For both the detection and discrimination animals, whisking frequencies during the initial sessions were typical of the basic $(6-9 \mathrm{~Hz})$ "exploratory" whisking pattern. The power spectra of the detection animals did not change over the course of learning in either the successful or the unsuccessful animals. However, the two successful object discrimination animals showed a shift to higher frequencies over the course of training, which was not seen in the unsuccessful rat. A significant association between bandwidth and successful discrimination performance has been reported in previous studies of texture discrimination (Carvell and Simons, 1995, 1996). Confirmation of that relationship for a different task and in head-fixed animals both extends the generality of the finding and suggests that our testing paradigms do not disrupt "normal" patterns of discriminative whisking.

Successful performance in the detection animals is associated with variations in the amount of whisking, 
manifested as a significant difference between the number of whisks emitted on $\mathrm{S}+$ and $\mathrm{S}-$ trials during the final phase of training. Interestingly, both successful animals show consistently more whisking during the $\mathrm{S}-$ trials, but no such difference is seen in the unsuccessful subject. The increased whisking on the $S-$ trials may reflect the utility of repeated sampling which would increase the likelihood of detection and decrease the probability of incorrect responses. The fact that none of the other kinematic variables, including frequency, were correlated with performance suggests that the rat's detection "strategy" involves modulation of the amount but not the type of whisking.

Successful performance on the object discrimination task was associated, not only with higher whisking frequencies, but with decreased mean durations of individual whisking movements, changes in protraction amplitude and increased whisking on $\mathrm{S}$ - trials during the last three sessions-a period when discrimination performance was at or approaching criterion levels. While discrimination performance did not seem to be associated with the absolute amplitudes of emitted whisks, the relative change in mean amplitude over the course of training was much greater for the two animals that met criterion than for the third, which did not $\left(7-10^{\circ}\right.$ vs $1^{\circ}$ ). These data strongly suggest that object discrimination requires modulation of both the amount and the type of whisking. The only kinematic variable which did not correlate significantly with task performance was velocity, although the two rats that achieved criterion performance showed a trend towards decreasing velocity over the course of training.

Because our primary focus was methodological, we did not examine the effects of systematically varying stimulus dimensions on the development of whisking movement patterns. Nevertheless, despite substantial differences in training paradigm, data recording and analysis we have confirmed the previous finding that successful discriminative behavior is associated with the presence of systematic variations in whisking movement patterns which may function as whisking "strategies" (Carvell and Simons, 1995).

\section{Functional characterization of whisking behavior: differential contributions of micro- and macrovibrissae}

Brecht et al. (1997) has noted that the different whisker types which make up the vibrissa array have often been treated as functionally equivalent. Using an ingenious testing paradigm, involving either localization of, or discrimination between small palatable and unpalatable cookies differing in shape, these investigators showed that the effects of removing either the micro- or macrovibrissae varied with task type. Macrovibrissa removal disrupted the localization, but not the recognition task. Microvibrissa removal disrupted the object recognition but not the localization task. Based upon these findings and differences in the topography and spatial density of the two classes of sensory hair, Brecht et al. (1997) concluded that the macrovibrissae function "as a distance detector array; the microvibrissae. .. as a high resolution tactile sensor" (1997, p. 97).

That the macrovibrissae contribute to spatial perception has been repeatedly demonstrated (Vincent, 1912; Schiffman et al., 1970; Hutson and Masterton, 1986; Diamond et al., 1999). The contribution of the microvibrissae to high-resolution discriminative behavior has been less convincingly documented. Brecht notes that "the object recognition deficit introduced by shaving is very transient-one day in most animals" (Brecht, pers. commun., 1998). Dissociation of the contributions of microvibrissae and snout skin afferents is also difficult. On the other hand, Carvell and Simons (1990, 1995) have shown that variations in the movement patterns of the large motile macrovibrissae are associated with the acquisition of high-resolution texture discriminations. Furthermore, the present study demonstrates that object detection and discrimination may be accomplished using only the macrovibrissae.

Identification of the "function" of a biological structure such as a sensory organ will depend upon the range and type of behavioral tasks used to "interrogate" that structure. Characterization of vibrissal function is complicated by substantial differences in task demands and testing conditions in the available studies (distal vs proximal stimuli; highvs low-resolution requirements). The use of a gapcrossing paradigm and distal stimuli obviously engages the macrovibrissae but does not exclude a contribution by inputs generated by lateral head movements across the microvibrissae. (Note: Carvell and Simons (1995) report that, during acquisition of the texture discrimination, the small rostral whiskers remain protracted and in contact with the discriminanda during active palpation with the large caudal whiskers (Carvell and Simons, 1990, p. 2641).) Use of an elevated stage or open field for an object recognition task involving proximal stimuli (Brecht et al., 1997, pers. commun.) may produce a bias towards the use of the microvibrissae. The paradigm used in the present study insures exclusive use of the macrovibrissae in a low-resolution discrimination task. We have no reports of performance on a highresolution discrimination task under conditions which insure exclusive use of either the macro- or microvibrissae. The currently available data suggest that both classes of vibrissa can support a variety of functions depending upon the behavioral context. The methods used in the present study may facilitate analysis of those functions.

\section{Acknowledgments}

This work was supported by Research Scientist Award MH-00320, by Grants MH-08366 and 
NS37263 and by PSC-CUNY Research Awards. We are grateful to Professor Peter Balsam (Barnard College) for his assistance in the development of the discrimination paradigm.

\section{References}

Bermejo, R., M. Harvey, P. Gao, and H.P. Zeigler (1996) Conditioned "whisking" in the rat. Somatosens Mot Res 13: 225-234.

Bermejo, R., D. Houben, and H.P. Zeigler (1998) Optoelectronic monitoring of individual whisker movements in rats. $\mathcal{F}$ Neurosci Meth 83: 89-96.

Bermejo, R., and H.P. Zeigler (2000) Technical Note: "Realtime" monitoring of vibrissa contacts during rodent whisking. Somatosens Mot Res 17: 309-314.

Brecht, M., B. PreIlowsi, and M.M. Merzenich (1997) Functional architecture of the mystacial vibrissae. Behav Brain Res 94: 81-97.

Carvelu, G.E., and D.J. Simons (1990) Biometric analyses of vibrissal tactile discrimination in the rat. $\mathcal{f}$ Neurosci 10: $2638-2648$.

Carveli, G.E., and D.J. Simons (1995) Task and subject related differences in sensorimotor behavior during active touch. Somatosens Mot Res 12: 1-9.

Carveli, G.E., and D.J. Simons (1996) Abnormal tactile experience early in life disrupts active touch. $\mathcal{f}$ Neurosci 15: $2750-2757$.

DehNHARDT, G. (1994) Tactile size discrimination by a California sea lion (Zalophus californianus) using its mystacial vibrissae. $\mathcal{F}$ Comp Physiol A 175: 791-800.

Dehnhardt, G., and G. Ducker (1996) Tactual discrimination of size and shape by a California sea lion (Zalophus californianus). Anim Learn Behav 24: 366-374.

Diamond, M., R. Petersen, and J. Harris (1999) Learning through maps: functional significance of topographic organization in the somatosensory cortex. F Neurobiol 41: 64-68.

DORFL, J. (1982) The musculature of the mystacial vibrissae of the white mouse. F Anat 142: 173-184.

Hutson, K.A., and R.B. MAsterton (1986) The sensory contribution of a single vibrissal cortical barrel. $\mathcal{F}$ Neurophysiol 56: 1196-1223.

LeDERMAN, S.J., and R. KLATZKY (1987) Hand movements: a window into haptic object recognition. Cognitive Psychol 19: 342-368.

MEssing, R.B. (1972) The sensitivity of rats to lights of different wavelengths. Vision Res 12: 753-761.

ROSENBERGER, B.P., and J.T. ERNEST (1971) Behavioral assessment of absolute visual thresholds in the albino rat.Vision Res 11: 199-207.

Schiffman, H.R., R. Lore, J. Passafiume, and R. Neeb (1970) Role of vibrissae for depth perception in the rat. Anim Behav 18: 290-292.

VINCENT, S.B. (1912) The function of the vibrissae in the behavior of the white rat. Behav Monog 1: 1-181.

WELKER, W.I. (1964) Analysis of sniffing of the albino rat. Behaviour 22: 223-244.

Willis, M.A., and E.A. Arbas (1997) Centrally patterned behavior generates sensory input for adaptive control. In Neurons, Networks and Motor Behavior, P.S.G. STEIN, S. Grillner, A.I. Selverston, and D.G. StuArt, eds., MIT Press, Cambridge, MA, pp. 269-275.

WINESKI, L.E. (1983) Facial morphology and vibrissal movement in the golden hamster. F Morphol 183: 199-217.

ZEIGLER, H.P., and L.B. WyCKOFF (1961) Observing responses and discrimination learning-set in the pigeon. $Q \mathcal{F}$ Exp Psychol 13: 129 . 